\title{
ITERATED FUNCTION SYSTEMS WITH OVERLAPS AND SELF-SIMILAR MEASURES
}

\author{
KA-SING LAU, SZE-MAN NGAI AND HUI RAO
}

\begin{abstract}
The paper considers the iterated function systems of similitudes which satisfy a separation condition weaker than the open set condition, in that it allows overlaps in the iteration. Such systems include the well-known Bernoulli convolutions associated with the PV numbers, and the contractive similitudes associated with integral matrices. The latter appears frequently in wavelet analysis and the theory of tilings. One of the basic questions is studied: the absolute continuity and singularity of the self-similar measures generated by such systems. Various conditions to determine the dichotomy are given.
\end{abstract}

\section{Introduction}

We will call a family of contractive maps $\left\{S_{j}\right\}_{j=1}^{N}$ on $\mathbb{R}^{d}$ an iterated function system (IFS). An iterated function system will generate an invariant compact subset $K=$ $\bigcup_{j=1}^{N} S_{j} K$, and if, further, the system is associated with a set of probability weights $\left\{w_{j}\right\}_{j=1}^{N}$, then it will generate an invariant measure

$$
\mu=\sum_{i=1}^{N} w_{j} \mu \circ S_{j}^{-1} .
$$

In order to obtain sharp results on the invariant set $K$ or the invariant measure $\mu$, it is often assumed that the maps are similitudes (or the extension to self-conformal maps). The corresponding $K$ and $\mu$ are called the self-similar set and the self-similar measure respectively. For the iteration, it is often assumed that the iterated function system satisfies the open set condition (OSC) [10]. One of the advantages of the open set condition is that the 'generic' points of the set $K$ can be uniquely represented in a symbolic space, and the dynamics of the iterated function system can be identified with the shift operation in the symbolic space. Without the open set condition, the iteration has overlaps; then such a representation will break down, and it is more difficult to handle the situation.

The simplest case of an iterated function system with overlaps is provided by the maps

$$
S_{1} x=\rho x, S_{2} x=\rho x+1, x \in \mathbb{R} \quad \text { with } \quad \frac{1}{2}<\rho<1 .
$$

The invariant measure $\mu$ associated with the weights $\frac{1}{2}$ has been studied for a long time in the context of Bernoulli convolutions [27]. Recently Solomyak [23, 28] proved

Received 18 September 1999; revised 18 February 2000.

2000 Mathematics Subject Classification 28A80 (primary), 42B10 (secondary).

The first author's research was partially supported by an HK RGC grant. The second author's research was partially supported by a postdoctoral fellowship from CUHK and by the NSF grant DMS-96-32032. The third author's research was partially supported by a postdoctoral fellowship from CUHK.

J. London Math. Soc. (2) 63 (2001) 99-116. (C) London Mathematical Society 2001. 
that for almost all such $\rho$, the measure $\mu$ is absolutely continuous. This solves a conjecture of Erdös. The 'transversality' argument used in [23] has also been used to consider a variety of iterated function systems with overlaps (by Peres and Solomyak [24], and by Peres and Schlag [22]). Other considerations on such systems with overlaps related to digit expansion can be found in $[\mathbf{1 1}, \mathbf{1 2}, \mathbf{2 5}, \mathbf{2 6}$.

In [17] Lau and Ngai introduced a weak separation condition (WSC) on an iterated function system of similitudes that has overlaps. This condition is weaker than the open set condition, and includes many of the important overlapping cases (see the examples in Section 2). In particular, it includes the system in equation (1.2) where $\rho^{-1}$ is a PV number. The multifractal structure of $\mu$ related to these numbers has been studied in detail in $[\mathbf{8}, \mathbf{9}, \mathbf{1 6}-\mathbf{1 8}]$.

In this paper we continue the study of the weak separation condition (see Definition 2.1). We will restrict our attention to similitudes $\left\{S_{j}\right\}_{j=1}^{N}$ on $\mathbb{R}^{d}$ with the same contraction ratio, that is,

$$
S_{j} x=A_{j} x+b_{j}=\rho R_{j} x+b_{j}
$$

where $0<\rho<1$ and $R_{j}$ is an orthogonal transformation. It is known that the selfsimilar measure $\mu$ is either absolutely continuous or continuously singular, but it is rather difficult to determine the dichotomy. Our main purpose in this paper is to study this problem. We prove the following theorem.

THEOREM 1.1. Let $\left\{S_{j}\right\}_{j=1}^{N}$ be an IFS as in equation (1.3) and assume that it satisfies the WSC. Suppose that $w_{j}>\rho^{d}$ for at least one $j$. Then the self-similar measure is singular.

For the proof of the theorem we observe that the weak separation condition implies that any ball $B_{\rho^{n}}(x)$ contains a (uniformly) bounded number of $S_{\sigma}\left(x_{0}\right),|\sigma|=n$. This property yields the key Proposition 2.3 which allows us to retain some control over counting the overlaps. We can then use it to handle the product measure on the symbolic space and the self-similar $\mu$ as its projection.

By using the Lebesgue density theorem and Theorem 1.1 we have the following interesting result.

THEOREM 1.2. Let $\left\{S_{j}\right\}_{j=1}^{N}$ be as above. If the self-similar measure $\mu$ is absolutely continuous, then the density function $f=D \mu \in L^{1}\left(\mathbb{R}^{d}\right)$ is actually in $L^{\infty}\left(\mathbb{R}^{d}\right)$.

The second theorem allows us to determine the absolute continuity by checking the existence of the $L^{2}$-density, which is simpler for self-similar measures. By assuming a slightly stronger condition on the iterated function system, which we call WSC* $^{*}$ (see $\S 4$ ), we can make use of the self-similar identity (1.1) and the correlation of $\mu$ to construct a nonnegative, irreducible transition matrix $T_{I}$ (where $I$ stands for the identity map on $\mathbb{R}^{d}$ ) and prove the following theorem.

THEOREM 1.3. Suppose that $\left\{S_{j}\right\}_{j=1}^{N}$ is an IFS as in equation (1.3) and satisfies the WSC*. Then $\mu$ is absolutely continuous if and only if

$$
\lambda_{\text {max }}=\rho^{d}
$$

where $\lambda_{\max }$ is the maximal eigenvalue of $T_{I}$. 
We will see that $\lambda_{\max } \geqslant \rho^{d}$ always holds (Proposition 4.4). When $\lambda_{\max }>\rho^{d}$, we can use $\lambda_{\text {max }}$ to determine the $L^{2}$-dimension of $\mu: \operatorname{dim}_{2}(\mu)=\left|\log \lambda_{\text {max }} / \log \rho\right|($ Theorem 4.1). The construction of the $T_{I}$ is quite direct, and can be implemented for the concrete cases.

We organize the paper as follows. We give the definition of the weak separation condition in Section 2, together with some examples and properties. Theorems 1.1 and 1.2 are proved in Section 3. In Section 4 we will define the transition matrix $T_{I}$ and prove Theorem 1.3. We also discuss some examples and the construction of the matrix $T_{I}$. To conclude the paper, we consider in Section 5 an extension of the classical Bernoulli convolution associated with the PV numbers, and prove the singularity in such a case. This extends a result of Erdös [27].

We remark that for the case $S_{j}(x)=\frac{1}{2}(x+j)$, where $j=0, \ldots, N$ on $\mathbb{R}$, if the $\mu$ in Theorem 1.3 is absolutely continuous and if we let $f=D \mu$ be the Radon Nikodym derivative of $\mu$, then the eigenvalue of $T_{I}$ with the second-largest magnitude can be used to determine the regularity of $f$. In other words, if we let

$$
\tilde{\lambda}=\max \left\{|\lambda|: \lambda \text { is an eigenvalue of } T_{I}, \lambda \neq \lambda_{\max }\right\},
$$

then the $L^{2}$-Lipschitz exponent of $f$ is given by $\frac{1}{2}(|(\log \tilde{\lambda}) /(\log 2)|-1)$. The result is a special case in [20]. The proof is more complicated than that of Theorem 1.3 because the corresponding eigenvalue and eigenvector may not be positive. We conjecture that the result is still true in the present setup of the weak separation condition.

\section{The weak separation condition}

Throughout the paper we assume that $S_{j}: \mathbb{R}^{d} \longrightarrow \mathbb{R}^{d}$, where $1 \leqslant j \leqslant N$, are contractive similitudes defined by

$$
S_{j} x=A_{j}\left(x+d_{j}\right)=A_{j} x+b_{j},
$$

where $b_{j}=A_{j} d_{j} \in \mathbb{R}^{d}, A_{j}=\rho R_{j}$ with $0<\rho<1$ and $R_{j}$ orthonormal matrices. We will use $\Sigma_{n}$ to denote the set of multi-indices $\sigma=\left(j_{1}, \ldots, j_{n}\right)$ and use $|\sigma|=n$ to denote the length of $\sigma$. Let $S_{\sigma}=S_{j_{1}} \circ \ldots \circ S_{j_{n}}$. Then

$$
\begin{aligned}
S_{\sigma}(x) & =A_{j_{1} \ldots j_{n}} x+A_{j_{1} \ldots j_{n-1}} b_{j_{n}}+A_{j_{1} \ldots j_{n-2}} b_{j_{n-1}}+\ldots+b_{j_{1}} \\
& =A_{j_{1} \ldots j_{n}} x+\sum_{i=1}^{n} A_{j_{1} \ldots j_{i-1}} b_{j_{i}} .
\end{aligned}
$$

In particular, if $A_{j}=A$ for all $j$ then $S_{\sigma}(x)=A^{n} x+\sum_{i=1}^{n} A^{i-1} b_{j_{i}}$.

Let $\left\{w_{j}\right\}_{j=1}^{N}$ be a set of probability weights associated with the iterated function system $\left\{S_{j}\right\}_{j=1}^{N}$, and let $\mu$ be the self-similar measure defined by $\mu=\sum_{j=1}^{N} w_{j} \mu \circ S_{j}^{-1}$ as in equation (1.1). For a fixed $x_{0}$, we define the discrete measure $\mu_{n}$ by

$$
\mu_{n}\{x\}=\sum\left\{w_{\sigma}: S_{\sigma}\left(x_{0}\right)=x,|\sigma|=n\right\}, \quad x \in \mathbb{R}^{d}
$$

where $w_{\sigma}=w_{j_{n}}$. It is well known that $\left\{\mu_{n}\right\}$ converges to $\mu$ weakly. Moreover, if we let $c=\max _{j}\left|b_{j}\right| /(1-\rho)$, then $\operatorname{supp} \mu$ is contained in a ball of radius $c$. For $x=$ $\sum_{i=1}^{\infty} A_{j_{1} \ldots j_{i-1}} b_{j_{i}}$, it is easy to show that

$$
\mu\left(B_{\rho^{n}}(x)\right) \leqslant \mu_{n}\left(B_{c \rho^{n}}(x)\right) \leqslant \mu\left(B_{2 c \rho^{n}}(x)\right)
$$

where $B_{r}(x)$ denotes the open ball of radius $r$ centered at $x$ [17]. If the iterated function system satisfies the open set condition, then the term $\mu_{n}\left(B_{r p}(x)\right)$ is quite easy to handle. In the following paragraph we define a condition on such a system that extends the open set condition and allows us to handle the term. 
Definition 2.1. We say that $\left\{S_{j}\right\}_{j=1}^{N}$ satisfies the weak separation condition (WSC) if there exists $x_{0} \in \mathbb{R}^{d}$ and a constant $a>0$ such that for $|\sigma|,\left|\sigma^{\prime}\right|=n$, then either

$$
S_{\sigma}\left(x_{0}\right)=S_{\sigma^{\prime}}\left(x_{0}\right) \quad \text { or } \quad\left|S_{\sigma}\left(x_{0}\right)-S_{\sigma^{\prime}}\left(x_{0}\right)\right| \geqslant a \rho^{n} .
$$

By a suitable translation we can assume that $x_{0}=0$. Also, note that the iterated function system is invariant on the subspace in $\mathbb{R}^{d}$ spanned by $S_{\sigma},|\sigma|=n, n \in \mathbb{N}$. Hence by restricting the iterated function system to the subspace, we can assume without loss of generality that for some $n$ large enough, $\left\{S_{\sigma}\left(x_{0}\right):|\sigma|=n\right\}$ spans $\mathbb{R}^{d}$. The definition says that after iterating $x_{0}$ by $\left\{S_{j}\right\}_{j=1}^{N}$ for $n$ times, all the points $S_{\sigma}\left(x_{0}\right),|\sigma|=n$ are either identical or separated by a distance $a \rho^{n}$. This definition was introduced in [17] under the more general setting that the similitudes can have different contraction ratios. Since for all the practical examples considered here, the maps in the iterated function system have the same contraction ratio, we just impose it in the definition for simplicity. The following proposition is immediate from the definition.

Proposition 2.1. Suppose that the IFS $\left\{S_{j}\right\}_{j=1}^{N}$ satisfies the WSC. Then any ball $B_{c \rho^{n}}(x)$ contains at most $\ell$ distinct $S_{\sigma}\left(x_{0}\right), \sigma \in \Sigma_{n}$.

It is also easy to see that condition (2.1) is equivalent to

$$
\text { either } S_{\sigma}^{-1} S_{\sigma^{\prime}}\left(x_{0}\right)=x_{0} \quad \text { or } \quad\left|S_{\sigma}^{-1} S_{\sigma^{\prime}}\left(x_{0}\right)-x_{0}\right| \geqslant a, \quad \forall|\sigma|=\left|\sigma^{\prime}\right| \text {. }
$$

In [4] Bandt and Graf showed that $\left\{S_{j}\right\}_{j=1}^{N}$ satisfies the open set condition if and only if there exists $x_{0} \in \mathbb{R}^{d}$ and $a>0$ such that $\left|S_{\sigma}^{-1} S_{\sigma^{\prime}}\left(x_{0}\right)-x_{0}\right| \geqslant a$ for all incomparable $\sigma$ and $\sigma^{\prime}$. Proposition 2.2 follows.

Proposition 2.2. If $\left\{S_{j}\right\}_{j=1}^{N}$ satisfies the open set condition, then it satisfies the WSC.

Our main interest is in iterated function systems that do not satisfy the open set condition. Below, we give a list of such examples with the weak separation condition.

ExAmple 2.1. Let $\left\{S_{j}\right\}_{j=1}^{N}$ be defined on $\mathbb{R}$ with $S_{j} x=1 / k x+b_{j}$ where $k \geqslant 2$ is an integer, and $b_{j}=c r_{j}$ with $c \in \mathbb{R}$ and $r_{j}$ rationals. We take $x_{0}=0$. Then for $\sigma=$ $\left(j_{1}, \ldots, j_{n}\right)$,

$$
S_{\sigma}(0)=c \sum_{i=1}^{n} \frac{r_{j_{i}}}{k^{i-1}}=\frac{c}{q} \sum_{i=1}^{n} \frac{t_{j_{i}}}{k^{i-1}}
$$

where $t_{j}=q r_{j}$, for $1 \leqslant j \leqslant N$, are integers. It is easy to see that the weak separation condition is satisfied by taking $a=c / q$ in the definition.

Note that the case with contraction ratio $\rho=1 / 2$ has been studied in great detail in wavelet theory in connection with the dilation equation

$$
f(x)=\sum_{j=1}^{N} c_{j} f(2 x-(j-1))
$$

The function $f$ can be considered as the density function of the corresponding absolutely continuous self-similar measure $\mu$ in equation (1.1), with $c_{j}=2 w_{j}$. In wavelet theory the $c_{j}$ may be negative but $\sum c_{j}$ must be 2 . 
Kenyon [12] and Rao and Wen [26] have studied the iterated function system for the contraction ratio $\rho=1 / 3$, with $N=3$. They are interested in the dimension of the invariant set $K$, and the tiling property related to the translation numbers $r_{j}$. Hu and Lau [9] and Fan et al. [5] have also given a detailed analysis of the multifractal structure of the self-similar measure generated by the iterated function system with such $\rho$.

Example 2.2. Let $\left\{S_{j}\right\}_{j=1}^{N}$ be defined on $\mathbb{R}$ with $S_{j} x=\rho x+b_{j}$ where $\beta=\rho^{-1}$ is a $\mathrm{PV}$ number, and $b_{j}=c r_{j}$ with $c \in \mathbb{R}$ and $r_{j}$ rationals. (Recall that $\beta>1$ is a PV number if it is an algebraic integer such that all its algebraic conjugates have modulus less than 1 (see [27]), for example, the golden ratio $(\sqrt{5}+1) / 2$.)

Similar to the above, we can write $S_{\sigma}(0)=c / q \sum_{i=1}^{n} t_{j_{i}} \rho^{i-1}$, for $t_{j_{i}} \in \mathbb{Z}$. The weak separation condition follows from a lemma of Garsia [6, Lemma 1.51]. The selfsimilar measure corresponding to $S_{1} x=\rho x$ and $S_{2} x=\rho x+(1-\rho)$ with weights $\frac{1}{2}$ each is the classical Bernoulli convolution [27]. The entropy dimension, $L^{p}$-spectrum, local dimension spectrum and the multifractal formalism of the Bernoulli convolution associated with the PV numbers, in particular with the golden ratio, have been studied in great detail by many authors (for example, 1, 2, 8, 14-17, 21).

In the above two examples the contraction ratios $\rho$ are algebraic integers. It is not difficult to construct an example with a more arbitrary $\rho$.

ExAmPle 2.3. Let $0<\rho<\frac{1}{3}, S_{1} x=\rho x, S_{2} x=\rho x+\rho$, and $S_{3} x=\rho x+1$, where $x \in \mathbb{R}$. Then $S_{1} \circ S_{3}(x)=S_{2} \circ S_{1}(x)=\rho^{2} x+\rho$. This implies that the open set condition is not satisfied according to the result of Bandt and Graf stated after equation (2.2). However, the iterated function system satisfies the weak separation condition: it is easy to show that

$$
S_{\sigma}(0)-S_{\sigma^{\prime}}(0)=\sum_{i=0}^{n-1} \rho^{i} \varepsilon_{i}, \quad \varepsilon_{i}=0, \pm \rho, \pm 1, \pm(1-\rho)
$$

can be written into the form $\sum_{i=0}^{n} \rho^{i} \eta_{i}$ where $\eta_{i}=0, \pm 1, \pm 2$. If $\sum_{i=0}^{n} \rho^{i} \eta_{i} \neq 0$ and $k$ is the smallest index such that $\eta_{k} \neq 0$, then since $0<\rho<\frac{1}{3}$,

$$
\left|S_{\sigma}(0)-S_{\sigma^{\prime}}(0)\right| \geqslant\left|\rho^{k}-2 \sum_{i=k+1}^{\infty} \rho^{i}\right| \geqslant\left(1-\frac{2 \rho}{1-\rho}\right) \rho^{k} \geqslant a \rho^{n}>0,
$$

where $a=(1-3 \rho) /(1-\rho)$.

EXAMPLE 2.4. In $\mathbb{R}^{d}$, we let $S_{j} x=A\left(x+d_{j}\right)$ where $B=A^{-1}$ is an integral expanding similarity matrix, with $d_{j} \in \mathbb{Z}^{d}$ and $d_{1}=0$. Then under a suitable norm on $\mathbb{R}^{d}, A$ is a contraction [13]. It is easy to show that $\left\{S_{j}\right\}_{j=1}^{N}$ satisfies the weak separation condition. Indeed, if we let $x_{0}=0$ and if $S_{\sigma}^{-1} S_{\sigma^{\prime}}(0) \neq 0$, then being an element in the integer lattice, $\left|S_{\sigma}^{-1} S_{\sigma^{\prime}}(0)\right| \geqslant 1$ and equation (2.2) applies.

This class of iterated function system has been studied in detail in connection with the theory of tiles under the more general setting using self-affine maps (see, for example, [13] and the references there). In the case of tiles, it is necessary to take $N=|\operatorname{det} B|$.

EXAmPLE 2.5. Let $A$ be as above, let $\Gamma$ be a finite group of integral matrices $\gamma$ with $\operatorname{det} \gamma= \pm 1$ and assume that $\Gamma$ satisfies $\Gamma A=A \Gamma$. Let $S_{j} x=A_{j}\left(x+d_{j}\right)$ where $A_{j}=$ $\gamma_{j} A, \gamma_{j} \in \Gamma, d_{j} \in \mathbb{Z}^{d}$. Then the above argument also implies that $\left\{S_{j}\right\}_{j=1}^{N}$ satisfies the weak 
separation condition. This class of iterated function system was used by Bandt [3] and $\mathrm{Xu}[30]$ to study tiles that involve rotations and reflections. For example, let

$$
A^{-1}=\left[\begin{array}{cc}
1 & -1 \\
1 & 1
\end{array}\right], \quad \Gamma=\left\{\left[\begin{array}{cc}
\varepsilon_{1} & 0 \\
0 & \varepsilon_{2}
\end{array}\right],\left[\begin{array}{cc}
0 & \varepsilon_{1} \\
\varepsilon_{2} & 0
\end{array}\right]: \varepsilon_{i}= \pm 1\right\}
$$

$S_{1} x=A x, S_{2} x=\gamma A x+\left[\begin{array}{l}1 \\ 0\end{array}\right]$ with $\gamma=\left[\begin{array}{cc}-1 & 0 \\ 0 & 1\end{array}\right]$. Then the corresponding invariant set

is the Lévy dragon [3].

To conclude this section we prove some useful consequences of the weak separation condition that will be needed in the following sections. In order to avoid confusion in the counting, we will identify $S_{\sigma}$ and $S_{\sigma^{\prime}},|\sigma|=\left|\sigma^{\prime}\right|=n$, if they are equal, and denote the set of such distinct $S_{\sigma}$ by $\mathscr{A}_{n}$. For $\sigma \in \Sigma_{n}$, we will use $[\sigma]$ to denote the equivalence class $\left\{\sigma^{\prime} \in \Sigma_{n}: S_{\sigma^{\prime}}=S_{\sigma}\right\}$.

Proposition 2.3. Suppose that $\left\{S_{j}\right\}_{j=1}^{N}$ satisfies the WSC. Then for any bounded subset $D \subset \mathbb{R}^{d}$, there exists $\gamma>0$ such that for any $x \in \mathbb{R}^{d}, n \in \mathbb{N}$,

$$
\#\left\{S \in \mathscr{A}_{n}: x \in S(D)\right\}<\gamma .
$$

Proof. Suppose that the proposition is false; then there exists a bounded $D$ such that for any $M>0$,

$$
\#\left\{S \in \mathscr{A}_{n}: x \in S(D)\right\}>M
$$

for some $n$ and $x$. By enlarging the set $D$ we can assume that the $x_{0}$ in the definition of the weak separation condition is in $D$ and that $\bigcup_{j=1}^{N} S_{j}(D) \subset D$. It follows that $\#\left\{S \in \mathscr{A}_{n}: S(D) \subseteq B_{\rho^{n}|D|}(x)\right\}>M$, and hence that

$$
\#\left\{S \in \mathscr{A}_{n}: S\left(x_{0}\right) \in B_{\rho^{n}|D|}(x)\right\}>M .
$$

Since $B_{\rho^{n}|D|}(x)$ contains at most $l$ distinct $S_{\sigma}\left(x_{0}\right),|\sigma|=n$ (see Proposition 2.1), there exists $s$ such that

$$
\#\left\{S \in \mathscr{A}_{n}: S\left(x_{0}\right)=s\right\}>\frac{M}{l} .
$$

Let $E_{s}=\left\{S \in \mathscr{A}_{n}: S\left(x_{0}\right)=s\right\}$. For $S_{\sigma}, S_{\sigma^{\prime}} \in E_{s}$, we have

$$
\begin{aligned}
S_{\sigma}(x)-S_{\sigma^{\prime}}(x) & =\left(A_{\sigma}\left(x-x_{0}\right)+S_{\sigma}\left(x_{0}\right)\right)-\left(A_{\sigma^{\prime}}\left(x-x_{0}\right)+S_{\sigma^{\prime}}\left(x_{0}\right)\right) \\
& =\left(A_{\sigma}-A_{\sigma^{\prime}}\right)\left(x-x_{0}\right)=\rho^{n}\left(R_{\sigma}-R_{\sigma^{\prime}}\right)\left(x-x_{0}\right) .
\end{aligned}
$$

Since $M$ can be arbitrarily large and there are at least $M$ distinct $S_{\sigma}$ in $\mathscr{A}_{n}$, we can choose distinct $R_{\sigma}, R_{\sigma^{\prime}}$ so that $\left\|R_{\sigma}-R_{\sigma^{\prime}}\right\|$ is arbitrarily small (depending on $M$ ).

We can assume without loss of generality that there exists $n_{0}$ such that $\left\{S_{\tau}\left(x_{0}\right):|\tau|=n_{0}\right\}$ contains 0 and spans $\mathbb{R}^{d}$ (see the remark after Definition 2.1). We claim that for any $\sigma, \sigma^{\prime} \in \Sigma_{n}$ with $S_{\sigma^{\prime}} \neq S_{\sigma}$, we have $S_{(\sigma, \tau)}\left(x_{0}\right) \neq S_{\left(\sigma^{\prime}, \tau\right)}\left(x_{0}\right)$ for at least one of the $\tau$. Otherwise, if we let $T=S_{\sigma^{\prime}}^{-1} S_{\sigma}$, then $S_{\tau}\left(x_{0}\right),|\tau|=n_{0}$ are fixed points of $T$. Recall that $\left\{S_{\tau}\left(x_{0}\right):|\tau|=n_{0}\right\}$ spans $\mathbb{R}^{d}$ and $T$ is an isometry of $\mathbb{R}^{d}$. This forces $T$ to be the identity map, which is a contradiction.

Now for any $a>0$, we choose $\sigma, \sigma^{\prime}$ with $S_{\sigma}, s_{\sigma}^{\prime} \in E_{s}$ such that

$$
\left\|R_{\sigma}-R_{\sigma^{\prime}}\right\| \leqslant a \rho^{n_{0}} \min \left\{\left\|S_{\tau}\left(x_{0}\right)-x_{0}\right\|^{-1}:|\tau|=n_{0}\right\} .
$$

Then for $\tau$ satisfying $S_{(\sigma, \tau)}\left(x_{0}\right) \neq S_{\left(\sigma^{\prime}, \tau\right)}\left(x_{0}\right)$ we have

$$
\begin{aligned}
0<\left\|S_{(\sigma, \tau)}\left(x_{0}\right)-S_{\left(\sigma^{\prime}, \tau\right)}\left(x_{0}\right)\right\| & =\left\|S_{\sigma} S_{\tau}\left(x_{0}\right)-S_{\sigma^{\prime}} S_{\tau}\left(x_{0}\right)\right\| \\
& =\rho^{n}\left\|\left(R_{\sigma}-R_{\sigma^{\prime}}\right)\left(S_{\tau}\left(x_{0}\right)-x_{0}\right)\right\| \leqslant a \rho^{\left(n+n_{0}\right)} .
\end{aligned}
$$

This contradicts the weak separation condition. 
Note that if the iterated function system satisfies the open set condition with an associated open set $U$, and if we take $D=U$, then the $\gamma$ in the above proposition is 1. In later applications we actually assume that

$$
D \text { is closed, } D^{\circ} \neq \varnothing, x_{0} \in D^{\circ} \text { and } \bigcup_{j=1}^{N} S_{j}(D) \subset D .
$$

We will call such $D$ a basic region. It follows that the invariant set $K$ is contained in $D$.

Corollary 2.1. Suppose that $\left\{S_{j}\right\}_{j=1}^{N}$ satisfies the WSC. Then there exists $\gamma>0$ such that $\# \mathscr{A}_{n} \leqslant \gamma \rho^{-d n}$.

Proof. Let $D$ be a basic region, and let $\gamma$ be as in Proposition 2.3. Then $\bigcup_{S \in \mathscr{A}_{n}} S(D) \subset D$ and $m(D)>0$ where $m$ is the Lebesgue measure. By the above proposition, we see that each $x \in D$ is covered by at most $\gamma$ of $S(D)$ with $S \in \mathscr{A}_{n}$. It follows that

$$
\sum_{S \in \mathscr{A}_{n}} m(S(D)) \leqslant \gamma m(D)
$$

The self-similarity implies that $m(S(D))=\rho^{d n} m(D)$. Therefore $\# \mathscr{A}_{n} \leqslant \gamma \rho^{-d n}$, as claimed.

Corollary 2.2. Suppose that $\left\{S_{j}\right\}_{j=1}^{N}$ satisfies the WSC. Let D be a basic region. Then for any $c>0$, there exists $c_{1}>0$ such that for any $n$ and $x$,

$$
\#\left\{S \in \mathscr{A}_{n}: S(D) \cap B_{c p^{n}}(x) \neq \varnothing\right\}<c_{1} .
$$

Proof. Let $r_{n}=c \rho^{n}+|D| \rho^{n}$. Then $S(D) \cap B_{c \rho^{n}}(x) \neq \varnothing$ implies that $S(D) \subset$ $B_{r_{n}}(x)$. Also, by Proposition 2.3, we know that every point is covered by at most $\gamma$ of the $S(D), S \in \mathscr{A}_{n}$. It follows that

Hence

$$
\begin{aligned}
\gamma \cdot m\left(B_{r_{n}}(x)\right. & \geqslant \sum_{S \in \mathscr{A _ { n }}}\left\{m(S(D)): S(D) \cap B_{c \rho^{n}}(x) \neq \varnothing\right\} \\
& =\rho^{d n} m(D) \cdot \#\left\{S \in \mathscr{A}_{n}: S(D) \cap B_{c \rho^{n}}(x) \neq \varnothing\right\} .
\end{aligned}
$$

$$
\#\left\{S \in \mathscr{A}_{n}: S(D) \cap B_{c \rho^{n}}(x) \neq \varnothing\right\} \leqslant \gamma\left(m(D) \rho^{d n}\right)^{-1} m\left(B_{r_{n}}(x)\right)
$$

and we can choose a bound $c_{1}$ for the last expression.

\section{A sufficient condition for singularity}

Let $\left\{S_{j}\right\}_{j=1}^{N}$ be an iterated function system with associated weights $\left\{w_{j}\right\}_{j=1}^{N}$ and let $\mu$ be the self-similar measure as defined in the last section. Our first theorem in this section gives a simple criterion for such a measure to be singular. First we will introduce some notations in the symbolic space. Let

$$
\Sigma=\left\{\bar{\sigma}=\left(j_{1}, j_{2}, \ldots\right): j_{i} \in\{1, \ldots, N\}\right\},
$$

and let $\Sigma_{n}$ be the set of $\sigma$ with length $n$. Let $\pi$ be the projection of $\Sigma$ to $\mathbb{R}^{d}$ defined by

$$
\pi(\bar{\sigma})=\bigcap_{n=1}^{\infty} S_{j_{1} \ldots j_{n}}(K), \quad \bar{\sigma}=\left(j_{1}, j_{2}, \ldots\right),
$$

where $K$ is the invariant set of the iterated function system. For $C_{\sigma}$ a cylinder set with base $\sigma \in \Sigma_{n}$, it is clear that $\pi\left(C_{\sigma}\right)=S_{\sigma}(K)$. We let $P$ be the probability measure on $\Sigma$, 
and $P_{n}$ the probability on $\Sigma_{n}$. For $\Lambda \subset \Sigma_{n}$, we will use the abbreviated notation $P(\Lambda)$ to denote $P_{n}(\Lambda)$; that is, $P(\Lambda)=P_{n}(\Lambda)=P\left(C_{\Lambda}\right)$, where $C_{\Lambda}$ is the cylinder set with base $\Lambda$. Note that $\mu=P \circ \pi^{-1}$, and for $\sigma \in \Sigma_{n}$,

$$
\mu\left(\pi\left(C_{\sigma}\right)\right)=P\left(C_{[\sigma]}\right)=\sum_{\sigma^{\prime} \in[\sigma]} w_{\sigma^{\prime}} .
$$

Lemma 3.1. Suppose that $\left\{S_{j}\right\}_{j=1}^{N}$ satisfies the WSC. For any $\Lambda \subseteq \Sigma_{n}$, we let

$$
\tilde{\Lambda}=\left\{\sigma \in \Lambda: \sum_{\sigma^{\prime} \in[\sigma] \cap \Lambda} w_{\sigma^{\prime}}>\frac{\rho^{d n}}{4 \gamma}\right\} .
$$

Then $P(\Lambda)>\frac{1}{2}$ implies that $P(\tilde{\Lambda})>\frac{1}{4}$.

Proof. By Corollary 2.1 we have $\# \mathscr{A}_{n} \leqslant \gamma \rho^{-d n}$. This implies that

$$
P(\Lambda \backslash \tilde{\Lambda})=\sum\left\{w_{\sigma}: \sigma \in \Lambda \backslash \tilde{\Lambda}\right\}=\sum_{[\sigma]} \sum_{\sigma^{\prime} \in[\sigma]}\left\{w_{\sigma^{\prime}}: \sigma^{\prime} \in \Lambda \backslash \tilde{\Lambda}\right\} \leqslant \# \mathscr{A}_{n} \cdot \frac{\rho^{d n}}{4 \gamma} \leqslant \frac{1}{4},
$$

and $P(\tilde{\Lambda})=P(\Lambda)-P(\Lambda \backslash \tilde{\Lambda})>1 / 2-1 / 4=1 / 4$

THEOREM 3.1. Suppose that $\left\{S_{j}\right\}_{j=1}^{N}$ satisfies the WSC, and suppose that $w_{j}>\rho^{d}$ for at least one $1 \leqslant j \leqslant N$. Then $\mu$ is singular.

Proof. Our aim is to choose, for any $\varepsilon>0$, a subset $E \subseteq \mathbb{R}^{d}$ such that $\mu(E) \geqslant \frac{1}{2}$ and $m(E)<\varepsilon$. Without loss of generality we assume that $w_{1}>\rho^{d}$. Let $D$ be a basic region as in the last section, and let $p \in \mathbb{N}$ be such that

Let

$$
4 \gamma m(D)\left(\frac{\rho^{d}}{w_{1}}\right)^{p}<\varepsilon
$$

$$
\begin{aligned}
\Lambda_{1} & =\Sigma_{1}=\{1, \ldots, N\}, \\
\tilde{\Lambda}_{1} & =\left\{\sigma \in \Lambda_{1}: w_{\sigma}>\frac{\rho^{d}}{4 \gamma}\right\}, \\
\Lambda_{1}^{*} & =\left\{(\sigma, 1, \ldots, 1) \in \Sigma_{1+p}: \sigma \in \tilde{\Lambda}_{1}\right\}, \\
E_{1} & =\bigcup\left\{S_{\sigma}(D): \sigma \in \Lambda_{1}^{*}\right\} .
\end{aligned}
$$

Then Lemma 3.1 implies that $P\left(\tilde{\Lambda}_{1}\right) \geqslant \frac{1}{4}$, so that $P\left(\Lambda_{1}^{*}\right) \geqslant \frac{1}{4} \omega_{1}^{p}$. Moreover, for $\sigma \in \Lambda_{1}^{*}$ we have

It follows that

$$
w_{\sigma}>\frac{\rho^{d}}{4 \gamma} w_{1}^{p}=\frac{\rho^{d(1+p)}}{4 \gamma}\left(\frac{w_{1}}{\rho^{d}}\right)^{p}>\frac{\rho^{d(1+p)}}{\varepsilon} m(D) .
$$

$$
m\left(E_{1}\right) \leqslant \rho^{d(1+p)} m(D) \cdot \#\left\{S_{\sigma}: \sigma \in \Lambda_{1}^{*}\right\} \leqslant \varepsilon \sum_{\sigma \in \Lambda_{1}^{*}} w_{\sigma} \leqslant \varepsilon P\left(\Lambda_{1}^{*}\right) .
$$

Suppose that we have chosen $\Lambda_{i}^{*} \subseteq \Sigma_{1+i p}$, and $E_{i}=\bigcup\left\{S_{\sigma}(D): \sigma \in \Lambda_{i}^{*}\right\}$, for $i=$ $1, \ldots, k-1$, such that

(i) $\Lambda_{i}^{*} \subseteq \Sigma_{1+i p}$ and $C_{\Lambda_{i}^{*}} \cap C_{\Lambda_{j}^{*}}=\varnothing$ for $i \neq j$;

(ii) $P\left(\Lambda_{i}^{*}\right) \geqslant \frac{1}{4} w_{1}^{p}$;

(iii) $m\left(E_{i}\right) \leqslant \varepsilon P\left(\Lambda_{i}^{*}\right)$. 
If $\sum_{i=1}^{k-1} P\left(\Lambda_{i}^{*}\right) \geqslant 1 / 2$, we stop the construction. Otherwise we let $\left.\sigma\right|_{n}$ denote the first $n$ coordinates of $\sigma$ and define

$$
\begin{aligned}
& \Lambda_{k}=\left\{\sigma \in \Sigma_{1+(k-1) p}:\left.\sigma\right|_{1+i p} \notin \Lambda_{i}^{*}, 1 \leqslant i \leqslant k-1\right\}, \\
& \tilde{\Lambda}_{k}=\left\{\sigma \in \Lambda_{k}: \sum_{\sigma^{\prime} \in[\sigma] \cap \Lambda} w_{\sigma^{\prime}}>\frac{\rho^{d}(1+(k-1) p)}{4 \gamma}\right\}, \\
& \Lambda_{k}^{*}=\left\{(\sigma, 1, \ldots, 1) \in \Sigma_{1+k p}: \sigma \in \tilde{\Lambda}_{k}\right\}, \\
& E_{k}=\bigcup\left\{S_{\sigma}(D): \sigma \in \Lambda_{k}^{*}\right\} .
\end{aligned}
$$

Then it is clear that $P\left(\Lambda_{k}\right)>\frac{1}{2}$ and $\Lambda_{k}^{*} \in \Sigma_{1+k p}$. That $C_{\Lambda_{i}^{*}} \cap C_{\Lambda_{k}^{*}}=\varnothing$ for $0 \leqslant i<k$ follows from the choice of $\Lambda_{k}$. Condition (ii) is a direct consequence of the construction of $\Lambda$ and $\Lambda_{k}^{*}$ and an application of Lemma 3.1. The proof of condition (iii) is similar to that of inequality (3.1).

In view of condition (ii), the process must stop at some finite step, say at $k$. Let $E=\bigcup_{i=1}^{k} E_{i}$. We recall that $D$ satisfies $\bigcup_{j=1}^{N} S_{j}(D) \subseteq D$, and the closedness of $D$ implies that $K \subseteq D$. Hence

$$
\pi\left(C_{\Lambda_{i}^{*}}\right)=\bigcup_{\sigma \in \Lambda_{i}^{*}} S_{\sigma}(K) \subseteq \bigcup_{\sigma \in \Lambda_{i}^{*}} S_{\sigma}(D)=E_{i} .
$$

This implies that $\pi\left(\bigcup_{i=1}^{k} C_{\Lambda_{i}^{*}}\right) \subseteq E$, and it follows that

$$
\mu(E)=P\left(\pi^{-1} E\right) \geqslant P\left(\bigcup_{i=1}^{k} C_{\Lambda_{i}^{*}}\right)=\sum_{i=1}^{k} P\left(C_{\Lambda_{i}^{*}}\right) \geqslant \frac{1}{2} .
$$

On the other hand, condition (iii) implies that

$$
m(E) \leqslant \sum_{i=1}^{k} m\left(E_{i}\right) \leqslant \varepsilon \sum_{i=1}^{k} P\left(C_{\Lambda_{i}^{*}}\right)<\varepsilon .
$$

The singularity of $\mu$ is proved.

We have an interesting consequence of the above theorem.

THEOREM 3.2. Suppose that $\left\{S_{j}\right\}_{j=1}^{N}$ satisfies the WSC. If $\mu$ is absolutely continuous, then the density function $f=D \mu$ will be bounded; that is, $f \in L^{\infty}\left(\mathbb{R}^{d}\right)$. Moreover, $f$ satisfies

$$
f(x)=\sum_{j=1}^{N} c_{j} f \circ S_{j}^{-1}(x), \quad x \in \mathbb{R}^{d},
$$

where $c_{j}=\left|\operatorname{det} A_{j}\right|^{-1} w_{j}$.

Proof. We need only to show that $f \in L^{\infty}\left(\mathbb{R}^{d}\right)$. Suppose otherwise; then for any large $M>0$, the Lebesgue density theorem implies that there exists a ball $B_{\rho^{n}}(x)$ such that

$$
\frac{\mu\left(B_{\rho^{n}}(x)\right)}{\rho^{d n}}=\frac{1}{\rho^{d n}} \int_{B_{\rho^{n}}(x)} f(t) d t>M .
$$

If we iterate $\left\{S_{j}\right\}_{j=1}^{N}$ for $n$ times, we obtain the family $\mathscr{A}_{n}$, which we will use to form a new iterated function system. For each $S=S_{\sigma} \in \mathscr{A}_{n}$, the associated weight is

$$
w_{S}=\sum\left\{w_{\sigma^{\prime}}: \sigma^{\prime} \in[\sigma]\right\} .
$$


The corresponding self-similar identity is $\mu=\sum_{S \in \mathscr{A}_{n}} w_{S} \mu \circ S^{-1}$. By equation (3.2) we have

$$
\sum_{S \in \mathscr{A}_{n}} w_{S} \mu \circ S^{-1}\left(B_{\rho^{n}}(x)\right)>M \rho^{d n} .
$$

Now we fix a basic region $D$; then $\mu$ is supported by $D$. Hence $S(D) \bigcap B_{\rho^{n}}(x)=\varnothing$ implies that $D \bigcap S^{-1}\left(B_{\rho^{n}}(x)\right)=\varnothing$, and thus $\mu\left(S^{-1}\left(B_{\rho^{n}}(x)\right)\right)=0$. In view of Corollary

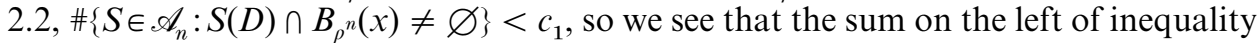
(3.3) has at most $c_{1}$ nonzero terms, and there exists at least one $w_{S}$ such that $w_{S}>$ $\left(M / c_{1}\right) \rho^{d n}$. We choose $M$ such that $M / c_{1}>1$. Then Theorem 3.1 implies that $\mu$ is singular. This is a contradiction.

Corollary 3.1. Suppose that $\left\{S_{j}\right\}_{j=1}^{N}$ satisfies the WSC. Then the self-similar measure $\mu$ is absolutely continuous if and only if the $L^{2}$-density of $\mu$ exists.

\section{The transition matrix}

Hardy and Littlewood [7] proved that for a probability measure $\mu$,

$$
\varlimsup_{h \rightarrow 0} \frac{1}{h^{2 d}} \int_{\mathbb{R}^{d}}\left|\mu\left(B_{h}(x)\right)\right|^{2} d x<\infty
$$

if and only if $\mu$ is absolutely continuous and $D \mu \in L^{2}$. There is no similar criterion for the absolute continuity of $\mu$ (with $D \mu \in L^{1}$ ). However, by using Corollary 3.1, we have the following proposition.

Proposition 4.1. Suppose that $\left\{S_{j}\right\}_{j=1}^{N}$ satisfies the WSC. Let $\beta$ be such that

$$
0<\varlimsup_{h \rightarrow 0} \frac{1}{h^{2 \beta}} \int_{\mathbb{R}^{d}}\left|\mu\left(B_{h}(x)\right)\right|^{2} d x<\infty .
$$

Then $\mu$ is absolutely continuous if and only if $\beta=d$.

In the following we will study the expression (4.1) and the exponent $\beta$ through certain transition matrix, to be defined. Note that the $L^{2}$-dimension (also called the correlation dimension) of $\mu$ is defined as

$$
\begin{aligned}
\underline{\operatorname{dim}}_{2}(\mu) & =\varliminf_{h \rightarrow 0} \frac{\log \int_{\mathbb{R}^{d}}\left|\mu\left(B_{h}(x)\right)\right|^{2} d x}{\log h}-d \\
& =\sup \left\{\alpha: \varlimsup_{h \rightarrow 0} \frac{1}{h^{d+\alpha}} \int_{\mathbb{R}^{d}}\left|\mu\left(B_{h}(x)\right)\right|^{2} d x<\infty\right\},
\end{aligned}
$$

and it follows that $\operatorname{dim}_{2}(\mu)=2 \beta-d$ (see [29]). The limit expression in expression (4.1) corresponding to the $\beta$ is called the upper mean quadratic variation of $\mu$ (see [19]). Similarly, we can define $\overline{\operatorname{dim}}_{2}(\mu)$ and $\operatorname{dim}_{2}(\mu)$.

Let $\mathscr{S}$ denote the set of maps $S=S_{\sigma}^{-1} S_{\sigma^{\prime}}$ for $\left(\sigma, \sigma^{\prime}\right) \in \bigcup_{n=1}^{\infty}\left(\Sigma_{n} \times \Sigma_{n}\right)$. We will consider $\mathscr{S}$ as a state space and define an (infinite) transition matrix on $\mathscr{S}$ as follows. For $S \in \mathscr{S}$, let

where

$$
T(S)=\sum_{S^{\prime} \in \mathscr{S}} w_{\left(S, S^{\prime}\right)} S^{\prime}
$$

$$
w_{\left(S, S^{\prime}\right)}=\sum_{i, j}\left\{w_{i} w_{j}: S_{i}^{-1} \circ S \circ S_{j}=S^{\prime}\right\}
$$


This amounts to saying that the transition from $S$ to $S^{\prime}$ has weight $w_{\left(S, S^{\prime}\right)}$. Also note that $\sum_{S^{\prime}} w_{\left(S, S^{\prime}\right)}=\left(\sum_{i} w_{i}\right)^{2}=1$. It follows that $T$ defines a Markov matrix on $\mathscr{S}$.

For a fixed $\beta$ and for any $S \in \mathscr{S}$, we define

$$
\Phi_{S}(h)=\frac{1}{h^{2 \beta}} \int_{\mathbb{R}^{a}} \mu\left(B_{h}(S x)\right) \mu\left(B_{h}(x)\right) d x .
$$

We use $\boldsymbol{\Phi}(h)$ to denote the vector $\left\{\Phi_{S}(h)\right\}_{S \in \mathscr{S}}$ and let $\langle\mathscr{S}\rangle$ denote the linear space spanned by $\mathscr{S}$. Then $\Phi_{S}(h)=\langle\boldsymbol{\Phi}(h), S\rangle$ and for any $v \in\langle\mathscr{S}\rangle$,

$$
\langle\boldsymbol{\Phi}(h), v\rangle=\sum_{S} v_{S} \Phi_{S}(h) .
$$

For convenience we also write the above expression as $\Phi_{v}(h)$. The following is the main purpose for defining the Markov matrix $T$ and the consideration of $\Phi_{S}(h)$.

Proposition 4.2. For $S \in \mathscr{S}, \Phi_{S}(h)=\rho^{d-2 \beta} \Phi_{T S}(h / \rho)$.

Proof. By substituting $\mu=\sum_{j} w_{j} \mu \circ S_{j}^{-1}$ into $\Phi_{S}(h)$, we have

$$
\begin{aligned}
\Phi_{S}(h) & =\frac{1}{h^{2 \beta}} \sum_{i, j} w_{i} w_{j} \int \mu\left(B_{h / \rho}\left(S_{i}^{-1} S(x)\right)\right) \mu\left(B_{h / \rho}\left(S_{j}^{-1}(x)\right)\right) d x \\
& =\frac{\rho^{d}}{h^{2 \beta}} \sum_{i, j} w_{i} w_{j} \int \mu\left(B_{h / \rho}\left(S_{i}^{-1} S S_{j}(x)\right)\right) \mu\left(B_{h / \rho}(x)\right) d x \\
& =\frac{\rho^{d}}{h^{2 \beta}} \sum_{S^{\prime}} w_{\left(S, S^{\prime}\right)} \int \mu\left(B_{h / \rho}\left(S^{\prime}(x)\right)\right) \mu\left(B_{h / \rho}(x)\right) d x \\
& =\rho^{d-2 \beta} \Phi_{T, S}\left(\frac{h}{\rho}\right) .
\end{aligned}
$$

We recall that $\operatorname{supp} \mu$ is contained in a ball of radius $(\rho /(1-\rho)) \max \left|d_{j}\right|$. We also recall that in the definition of the weak separation condition we can take $x_{0}=0$ without loss of generality.

Definition 4.2. Let $S_{j}(x)=A_{j}\left(x+d_{j}\right)$, where $j=1, \ldots, N$, with $A_{j}=\rho R_{j}$ as before. Let $\tilde{C}=(2 \rho /(1-\rho)) \max _{j}\left|d_{j}\right|$, and let

$$
\tilde{\mathscr{S}}=\{S \in \mathscr{S}:|S(0)| \leqslant \tilde{C}\} .
$$

We say that $\left\{S_{j}\right\}_{j=1}^{N}$ satisfies the weak separation condition* (WSC*) if $\tilde{\mathscr{S}}$ is a finite set.

We remark that from the definition of the WSC*, the set $\{S(0): S \in \tilde{\mathscr{S}}\}$ is a finite set, so there exists $a>0$ such that for $S, S^{\prime} \in \tilde{\mathscr{S}}$ with $S(0) \neq S^{\prime}(0)$, then $\left|S(0)-S^{\prime}(0)\right| \geqslant$ $a>0$. This makes the WSC* stronger than the WSC, which only requires that $|S(0)| \geqslant a$ for any $S \in \tilde{\mathscr{S}}$ with $S(0) \neq 0$ (see equation (2.2)).

We also remark that all the examples in Section 2 satisfy the WSC*. We will discuss this in more detail at the end of the section.

Proposition 4.3. Suppose that $\left\{S_{j}\right\}_{j=1}^{N}$ satisfies the WSC*. Then

(i) $T$ maps $\mathscr{S} \backslash \tilde{\mathscr{S}}$ to itself;

(ii) There exists $h_{0}>0$ such that for $0<h<h_{0}$ and $S \in \mathscr{S} \backslash \tilde{\mathscr{S}}, \Phi_{S}(h)=0$. 
Proof. (i) We let $S=S_{\sigma}^{-1} S_{\sigma^{\prime}} \in \mathscr{S} \backslash \tilde{\mathscr{S}}$, then $|S(0)|>\tilde{C}+\delta$ for some $\delta>0$. It follows that

$$
\begin{aligned}
\left|S_{i}^{-1} S S_{j}(0)\right| & \geqslant \rho^{-1}\left|S S_{j}(0)\right|-\left|d_{i}\right| \geqslant \rho^{-1}\left|S(0)+\rho R_{\sigma}^{-1} R_{\sigma^{\prime}}\left(R_{j} d_{j}\right)\right|-\max _{k}\left|d_{k}\right| \\
& \geqslant \frac{2 \rho}{(1-\rho)} \max _{k}\left|d_{k}\right|+\frac{\delta}{\rho}=\tilde{C}+\frac{\delta}{\rho} .
\end{aligned}
$$

(ii) In view of the above fact that $|S(0)|>\tilde{C}+\delta$ implies that $\left|S_{i}^{-1} S S_{j}(0)\right|>\tilde{C}+\rho^{-1} \delta$ and the hypothesis that $\tilde{\mathscr{S}}$ is a finite set, we can choose $h_{0}$ small enough so that for $S \notin \tilde{\mathscr{S}},|S(0)|>\tilde{C}+2 h_{0}$. We claim that for such $S, \mu\left(B_{h}(S x)\right) \mu\left(B_{h}(x)\right)=0$ for all $x$. Otherwise, there exist $x$ and $h$ such that $\mu\left(B_{h}(S x)\right) \neq 0$. By observing that the support of $\mu$ is continuous in $\tilde{C} / 2$, we have $|x| \leqslant \tilde{C} / 2+h$. It follows that

$$
|S(x)|=\left|S(0)+R_{\sigma}^{-1} R_{\sigma^{\prime}}(x)\right|>\left(\tilde{C}+2 h_{0}\right)-\left(\frac{\tilde{C}}{2}+h_{0}\right)>\frac{\tilde{C}}{2}+h_{0}
$$

and $\mu\left(B_{h}(S x)\right)=0$ for $h \leqslant h_{0}$. This is a contradiction, and part (ii) follows from the claim.

From the above proposition we can write $T$ as

$$
T=\left(\begin{array}{cc}
\tilde{T} & 0 \\
Q & T^{\prime}
\end{array}\right)
$$

where $\tilde{T}$ is a sub-Markov matrix on the states $\tilde{\mathscr{S}}$ (since the sum of each column of $T$ is 1 , the sum of each column of $\tilde{T}$ is $\leqslant 1$ ). $\tilde{T}$ is a finite matrix by the WSC*.

Let $\lambda$ be an eigenvalue of $\tilde{T}$ and $v$ a corresponding eigenvector. By Propositions 4.2 and 4.3 , we have

$$
\begin{aligned}
\Phi_{v}(h) & =\rho^{d-2 \beta} \Phi_{T v}\left(\frac{h}{\rho}\right)=\rho^{d-2 \beta}\left(\Phi_{\tilde{T} v}\left(\frac{h}{\rho}\right)+\Phi_{Q v}\left(\frac{h}{\rho}\right)\right) \\
& =\rho^{d-2 \beta}\left(\Phi_{\tilde{T} v}\left(\frac{h}{\rho}\right)+0\right)=\rho^{d-2 \beta} \Phi_{\lambda v}\left(\frac{h}{\rho}\right)=\lambda \rho^{d-2 \beta} \Phi_{v}\left(\frac{h}{\rho}\right) .
\end{aligned}
$$

If the matrix $\tilde{T}$ is irreducible, the Perron-Frobenius theorem implies that the maximal eigenvalue $\lambda_{\max }$ is positive, and that all the coordinates of the eigenvector $v$ are positive. If we take $\beta$ such that $\lambda_{\max } \rho^{d-2 \beta}=1$, then $\Phi_{v}(h)=\Phi_{v}(h / \rho)$. It is easy to show that $\Phi_{v}(h)>0$ and $0<\underline{\lim }_{h \rightarrow 0} \Phi_{v}(h) \leqslant \varlimsup_{\lim _{h \rightarrow 0}} \Phi_{v}(h)<\infty$. From Proposition 4.1 we see that the absolute continuity criterion is $\lambda_{\max }=\rho^{d}$.

However the matrix $\tilde{T}$ is not always irreducible. Hence we cannot guarantee that $\Phi_{v}(h)>0$, and we will need a little more elaborate work. We need to pick up the essential part of $\tilde{T}$ first. Let $I$ be the identity map in $\tilde{\mathscr{S}}$. Let $\mathscr{S}_{I}$ be the $\tilde{T}$-irreducible component of $\tilde{\mathscr{S}}$ that contains $I$; that is,

$S \in \mathscr{T}_{I}$ if and only if there exist $m, n \geqslant 1$ such that $w_{(I, S)}^{(m)}, w_{(S, I)}^{(n)}>0$

where $w_{\left(S, S^{\prime}\right)}^{(n)}$ denotes the $\left(S, S^{\prime}\right)$ entry of $\tilde{T}^{n}$. Let $T_{I}$ be the truncated square matrix of $\tilde{T}$ on $\mathscr{S}_{I}$; then $T_{I}$ is irreducible and is a finite matrix by the WSC*.

Theorem 4.1. Suppose that $\left\{S_{j}\right\}_{j=1}^{N}$ satisfies the WSC*. Let $\lambda_{\max }$ be the maximal eigenvalue of $T_{I}$ and let

$$
\beta=\frac{1}{2}\left(d+\left|\frac{\log \lambda_{\max }}{\log \rho}\right|\right) .
$$


Then the mean quadratic variation satisfies

$$
0<\varliminf_{h \rightarrow 0} \frac{1}{h^{2 \beta}} \int_{\mathbb{R}^{d}}\left|\mu\left(B_{h}(x)\right)\right|^{2} d x \leqslant \varlimsup_{h \rightarrow 0} \frac{1}{h^{2 \beta}} \int_{\mathbb{R}^{d}}\left|\mu\left(B_{h}(x)\right)\right|^{2} d x<\infty .
$$

The $L^{2}$-dimension of $\mu$ is hence given by $\operatorname{dim}_{2}(\mu)=\left|\log \lambda_{\max } / \log \rho\right|$.

As a direct consequence we have the following corollary.

Corollary 4.1. Suppose that $\left\{S_{j}\right\}_{j=1}^{N}$ satisfies the hypotheses of Theorem 4.1. Then $\mu$ is absolutely continuous if and only if $\lambda_{\max }=\rho^{d}$.

The theorem is a direct consequence of the following Lemmas 4.1 and 4.2. We first observe that the $(I, I)$ entry of $T_{I}^{n}$ is

$$
w_{(I, I)}^{(n)}=\sum_{\sigma, \sigma^{\prime} \in \Sigma_{n}}\left\{w_{\sigma} w_{\sigma^{\prime}}: S_{\sigma}^{-1} S_{\sigma^{\prime}}=I\right\}
$$

and $w_{(I, I)}^{(n)}=\sum_{S \in \mathscr{O}_{n}} w_{S}^{2}$. Since $T_{I}$ is a nonnegative irreducible matrix, it is well known that there exist $a_{1}, a_{2}>0$ such that

$$
a_{1} \lambda_{\max }^{n} \leqslant w_{(I, I)}^{(n)} \leqslant a_{2} \lambda_{\max }^{n} .
$$

Proposition 4.4. Under the assumption of Theorem 4.1, we have $\lambda_{\max } \geqslant \rho^{d}$.

Proof. By Corollary 2.1, we have $\# \mathscr{A}_{n} \leqslant \gamma \rho^{-d n}$. Since $\sum_{S \in \mathscr{A}_{n}} w_{S}=1$, the CauchySchwartz inequality implies that

$$
w_{(I, I)}^{(n)}=\sum_{S \in \mathscr{O}_{n}} w_{S}^{2} \geqslant \gamma^{-1} \rho^{d n} .
$$

Hence by inequality (4.2) we conclude that $\lambda_{\max } \geqslant \rho^{d}$.

Lemma 4.1. Under the assumption of Theorem 4.1 and letting $D$ be a basic region, there exists $c>0$ such that

$$
\int_{\mathbb{R}^{d}}\left|\mu\left(B_{|D| \rho^{n}}(x)\right)\right|^{2} d x \geqslant c \rho^{n d} \lambda_{\max }^{n}
$$

Proof. Let $h=|D| \rho^{n}$. For $S \in \mathscr{A}_{n}$, if $x \in S(D)$, then $S(D) \subset B_{h}(x)$. It follows that

$$
\int_{S(D)}\left|\mu\left(B_{h}(x)\right)\right|^{2} d x \geqslant \int_{S(D)}|\mu(S(D))|^{2} d x \geqslant \int_{S(D)} w_{S}^{2} d x=m(D) \rho^{d n} w_{S}^{2} .
$$

Since the WSC* implies the WSC, we see that each point is covered by at most $\gamma$ of the $S(D)$ (by Proposition 2.3). Hence

$$
\int_{\mathbb{R}^{d}}\left|\mu\left(B_{h}(x)\right)\right|^{2} d x \geqslant \frac{1}{\gamma} \sum_{S \in \mathscr{S}_{n}} \int_{S(D)}|\mu(S(D))|^{2} d x \geqslant \frac{m(D)}{\gamma} \rho^{d n} \sum_{S \in \mathscr{O}_{n}} w_{S}^{2}=c \rho^{n d} w_{(I, I)}^{(n)}
$$

where $c=m(D) / \gamma$. This, together with inequality (4.2), implies the lemma.

LEMmA 4.2. Under the same assumption as in Theorem 4.1 and for $\rho^{n+1} \leqslant h<\rho^{n}$, we have

$$
\int_{\mathbb{R}^{d}}\left|\mu\left(B_{h}(x)\right)\right|^{2} d x<c \rho^{d n} \lambda_{\max }^{n},
$$

for some $c>0$ independent of $n$. 
Proof. Let $Q_{j}$ be a $\rho^{n}$-mesh cube and let $\tilde{Q}_{j}=\bigcup_{x \in Q_{j}} B_{h}(x)$. Let $D(\supseteq K)$ be a basic region as before. Then by using $\mu=\sum_{S \in \mathscr{S}_{n}} w_{S} \mu \circ S^{-1}$, we have for $x \in Q_{j}$,

$$
\mu\left(B_{h}(x)\right) \leqslant \sum_{S \in \mathscr{A}_{n}}\left\{w_{S}: D \cap S^{-1}\left(B_{h}(x)\right) \neq \varnothing\right\} \leqslant \sum_{S \in \mathscr{A}_{n}}\left\{w_{S}: S(D) \cap \tilde{Q}_{j} \neq \varnothing\right\} .
$$

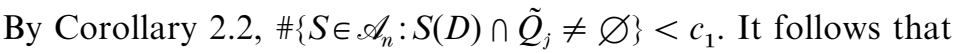

$$
\begin{aligned}
\int_{Q_{j}} \mid \mu( & \left.B_{h}(x)\right)\left.\right|^{2} d x \\
& \leqslant\left(\sum_{S \in \mathscr{O}_{n}}\left\{w_{S}: S(D) \cap \tilde{Q}_{j} \neq \varnothing\right\}\right)^{2} \cdot m\left(Q_{j}\right) \\
& \leqslant c_{1} \sum_{S \in \mathscr{O}_{n}}\left\{w_{S}^{2}: S(D) \cap \tilde{Q}_{j} \neq \varnothing\right\} \cdot m\left(Q_{j}\right) \quad \text { (Cauchy-Schwartz inequality) } \\
& =c_{2} \rho^{d n} \sum_{S \in \mathscr{A}_{n}}\left\{w_{S}^{2}: S(D) \cap \tilde{Q}_{j} \neq \varnothing\right\} .
\end{aligned}
$$

For each $S \in \mathscr{A}_{n}$, it is clear that $\#\left\{Q_{j}: S(D) \cap \tilde{Q}_{j} \neq \varnothing\right\}<c_{3}$ for some fixed $c_{3}$. Summing both sides of the above expressions, we obtain

$$
\int_{\mathbb{R}^{d}}\left|\mu\left(B_{h}(x)\right)\right|^{2} d x \leqslant c_{2} c_{3} \rho^{d n} \sum_{S \in \mathscr{A}_{n}} w_{S}^{2}=c_{4} \rho^{d n} w_{(I, I)}^{(n)} .
$$

The lemma now follows from expression (4.2).

To conclude this section we will discuss the examples in Section 2 in regard to the WSC* ${ }^{*}$. We show that the state space $\tilde{\mathscr{S}}$ and the matrix $\tilde{T}$ can easily be implemented after some concrete identifications.

Let $\left\{S_{j}\right\}_{j=1}^{N}$ be an iterated function system on $\mathbb{R}$ with $S_{j}(x)=\rho\left(x+d_{j}\right)$, where $0<$ $\rho<1$. Without loss of generality we assume that $0=d_{1}<d_{2} \ldots<d_{N}$. We can prove by induction that the state $S=S_{\sigma}^{-1} S_{\sigma^{\prime}} \in \mathscr{S}$ has the form

$$
S x=x+s, x \in \mathbb{R}
$$

for some $s \in \mathbb{R}$. We can represent the map $S$ by the translation number $s$. We construct the set $\mathscr{S}$ inductively, starting from $s=0$, by letting

$$
s^{\prime}=\rho^{-1} s+d_{j}-d_{i}, \quad 1 \leqslant i, j \leqslant N .
$$

The set $\tilde{\mathscr{S}}$ can be obtained by keeping those $s^{\prime}$ with $\left|s^{\prime}\right| \leqslant \tilde{C}=(2 \rho /(1-\rho)) d_{N}$. The matrix $T$ will send $s$ into the states $s^{\prime}$ in equation (4.3) with weight

$$
w_{\left(s, s^{\prime}\right)}=\sum\left\{w_{i} w_{j}: \rho^{-1} s+d_{j}-d_{i}=s^{\prime}\right\} .
$$

It can be checked from this that Examples 2.1-2.3 have the WSC* (for Example 2.2, we need to use Garsia's lemma again). For the numerical examples the reader can check on $[5, \mathbf{1 5}, \mathbf{1 8}]$. In all those cases $\tilde{T}=T_{I}$ and $\tilde{T}$ can be reduced further to smaller size by the symmetry of the $\tilde{\mathscr{S}}$.

For the iterated function system in Example 2.4, $S_{j}(x)=A\left(x+d_{j}\right)$ in $\mathbb{R}^{d}$, the maps $S \in \mathscr{S}$ still have the form $S x=x+s$. The WSC* is clear and the construction of the set $\tilde{\mathscr{S}}$ and the map $\tilde{T}$ is the same as the above one-dimensional case.

For the iterated function system in Example 2.5, we can prove by induction that each $S=S_{\sigma}^{-1} S_{\sigma^{\prime}} \in \mathscr{S}$ can be represented as

$$
S(x)=\gamma(x)+s,
$$

where $\gamma=A_{\sigma}^{-1} A_{\sigma^{\prime}} \in \Gamma$ and $s=S_{\sigma}^{-1} S_{\sigma^{\prime}}(0) \in \mathbb{Z}^{d}$. Hence each $S \in \mathscr{S}$ can be represented by 
$(\gamma, s)$. It is immediate to see that the system satisfies the $\mathrm{WSC}^{*}$ since $\Gamma$ is a finite set and $s \in \mathbb{Z}^{d}$.

For the construction of $\tilde{\mathscr{S}}$ and $\tilde{T}$, we first define $\gamma^{*}$ by $\gamma A=A \gamma^{*}$ and then observe that

$$
\begin{aligned}
S_{i}^{-1} S S_{j}(0) & =A_{i}^{-1}\left(\gamma A_{j} d_{j}+s\right)-d_{i} \\
& =\left(\gamma_{i}^{-1} \gamma \gamma_{j}\right)^{\#} d_{j}+A_{i}^{-1} s-d_{i} \\
& =\left(\gamma_{i}^{-1} \gamma \gamma_{j}\right)^{\#} d_{j}+S_{i}^{-1}(s) .
\end{aligned}
$$

Hence the set $\mathscr{S}$ can be constructed inductively as follows, starting from $(\gamma, s)=$ $(I, 0)$,

$$
\left(\gamma^{\prime}, s^{\prime}\right)=\left(\left(\gamma_{i}^{-1} \gamma \gamma_{j}\right)^{\#},\left(\gamma_{i}^{-1} \gamma \gamma_{j}\right)^{\#} d_{j}+S_{i}^{-1}(s)\right), \quad 1 \leqslant i, j \leqslant N .
$$

The set $\tilde{\mathscr{S}}$ is obtained by choosing the $(\gamma, s) \in \mathscr{S}$ such that $\left(\gamma_{i}^{-1} \gamma \gamma_{j}\right)^{\#} d_{j}+S_{i}^{-1}(s) \leqslant \tilde{C}$ in the construction. The map $T$ will send $(\gamma, s)$ into the above states with weight

$$
w_{\left((\gamma, s),\left(\gamma^{\prime}, s^{\prime}\right)\right)}=\sum_{i, j}\left\{w_{i} w_{j}:\left(\gamma_{i}^{-1} \gamma \gamma_{j}\right)^{\#}=\gamma^{\prime},\left(\gamma_{i}^{-1} \gamma \gamma_{j}\right)^{\#} d_{j}+S_{i}^{-1}(s)=s^{\prime}\right\} .
$$

\section{The case of $P V$ numbers}

Erdös proved that if $X=\sum_{i=1}^{\infty} \rho^{n} X_{n}$ where $\left\{X_{n}\right\}_{n=1}^{\infty}$ are independent identically distributed Bernoulli random variables and $1<\rho^{-1}<2$ is a PV number, then the distributional measure $\mu$ is singular [27]. In our present notation, $\mu$ is the self-similar measure

$$
\mu=\frac{1}{2} \mu \circ S_{1}^{-1}+\frac{1}{2} \mu \circ S_{2}^{-1}
$$

where $S_{1} x=\rho x$, and $S_{2} x=\rho x+1$. In the following we will extend Erdös' theorem to $S_{j}(x)=\rho x+b_{j}, 1 \leqslant j \leqslant N$, where $\rho^{-1}>1$ is a PV number and $b_{j}$ are rationals, and the probability weights are arbitrary (even negative). We need two technical lemmas.

For fixed $\left(k_{0}, n_{0}\right) \in \mathbb{Z} \times \mathbb{Z}$, consider the following relation:

$$
k \beta^{n}+k_{0} \beta^{n_{0}} \in \mathbb{Z} .
$$

If $k, n, p \in \mathbb{Z}$ satisfy $k \beta^{n}+k_{0} \beta^{n_{0}}=p$, we say that $(k, n, p)$ is a solution of relation (5.1); we also say that $(k, n)$ is a solution of relation $(5.1)$ if $(k, n, p)$ is a solution for some $p \in \mathbb{Z}$.

Lemma 5.1. Suppose that $\beta>1$ is an algebraic number and that at least one of its conjugate roots has modulus $<1$. Then for any $\left(k_{0}, n_{0}\right) \in \mathbb{Z} \times \mathbb{Z}$ with $k_{0}, n_{0} \neq 0$, the number of solutions of equation (5.1) is finite.

Proof. We first observe that $n \neq 0$, for otherwise $\beta^{n_{0}}=(p-k) / k_{0}$ will imply that $\beta$ and its conjugates are multiples of unity. This contradicts that $\beta>1$ and one of its conjugates has modulus $<1$. Next, if $(k, n, p)$ and $\left(k^{\prime}, n^{\prime}, p\right)$ are two distinct solutions of relation (5.1), then $\beta^{n-n^{\prime}}=k^{\prime} / k$ which is impossible for the same reason.

Let $\lambda$ be a conjugate of $\beta$ such that $|\lambda|<1$. If relation (5.1) has infinitely many solutions (for different $p$ ), then we have solutions $(k, n, p)$ with $|p|$ arbitrarily large. Hence

$$
\left|\frac{\beta}{\lambda}\right|^{n}=\frac{\left|k \beta^{n}\right|}{\left|k \lambda^{n}\right|}=\frac{\left|p-k_{0} \beta^{n_{0}}\right|}{\left|p-k_{0} \lambda^{n_{0}}\right|} \rightarrow 1,
$$

when $|p| \rightarrow \infty$. This contradicts the fact that $|\beta / \lambda|^{n}>|\beta / \lambda|>1$ when $n>0$, and $|\beta / \lambda|^{n}<|\beta / \lambda|^{-1}<1$ when $n<0$. 
LEMMA 5.2. Let $Q(\xi)=\sum_{j=1}^{N} c_{j} e^{2 \pi i b_{j} \xi}$ be a trigonometric polynomial with $\sum_{j=1}^{N} c_{j} \neq 0, c_{j} \in \mathbb{R}$ and $b_{j}$ rationals. Let $B$ be such that $B_{j}=B b_{j}$, where $1 \leqslant j \leqslant N$, are integers, and let $\beta$ be an algebraic number as in Lemma 5.1. Then there exists $m \in \mathbb{Z}$ such that $Q\left(m B \beta^{k}\right) \neq 0$ for all $k \in \mathbb{Z}$.

Proof. Let $\tilde{Q}(x)=\sum_{j=1}^{N} c_{j} x^{B_{j}}$ and let $\left\{x_{1}, \ldots, x_{s}\right\}$ be the roots of $\tilde{Q}(x)$ with modulus 1 that can be written as $e^{2 \pi i k_{\ell} \beta^{n} \ell}$, where $1 \leqslant \ell \leqslant s$. Then $\sum_{j=1}^{N} c_{j} \neq 0$ implies that $k_{l}$ and $n_{t} \neq 0$ for all $1 \leqslant l \leqslant s$. Note that $Q\left(m B \beta^{k}\right)=0$ if and only if $e^{2 \pi i m \beta^{k}}$ is a root of $\tilde{Q}$. It follows that

$$
m \beta^{k}-k_{\ell} \beta^{n_{\iota} \in \mathbb{Z}}
$$

for some $1 \leqslant \ell \leqslant s$. By Lemma 5.1 there are only finitely many $m$ for equation (5.2) to hold. Hence we can choose $m \in \mathbb{Z}$ such that $Q\left(m B \beta^{k}\right) \neq 0$ for all $k \in \mathbb{Z}$.

THEOREM 5.1. Let $\rho^{-1}$ be an irrational $P V$ number, let $b_{j}$ be rationals, and let

$$
S_{j} x=\rho x+b_{j}, \quad j=1, \ldots, N .
$$

Then for any set of probability weights $\left\{w_{j}\right\}_{j=1}^{N}$ the self-similar measure $\mu$ is singular.

Proof. By equation (1.1), the Fourier transformation $\Phi(\xi)=\hat{\mu}(\xi)$ satisfies

$$
\Phi(\xi)=Q(\xi) \Phi(\rho \xi)=\prod_{j=0}^{\infty} Q\left(\rho^{j} \xi\right)
$$

where $Q(\xi)=\sum_{j=1}^{N} w_{j} e^{2 \pi i b_{j} \xi}$. Note that $\Phi(0)=1$ and the product converges to $\Phi$ uniformly on compact subsets of $\mathbb{R}$. We will show that $|\Phi(\xi)|$ does not converge to 0 as $|\xi| \rightarrow \infty$. The Riemann-Lebesgue lemma will imply that $\mu$ is singular.

Let $\beta=\rho^{-1}$. We assume without loss of generality that there exists $B$ such that $B_{j}=B b_{j}$ are integers and $Q\left(B \beta^{k}\right) \neq 0$ for all $k \in \mathbb{Z}$ (Lemma 5.2). Let $C=\prod_{n=0}^{\infty} Q\left(B \beta^{-n}\right)$. Then $C \neq 0$. For $k>0$,

$$
\left|\Phi\left(B \beta^{k}\right)\right|=\prod_{n=0}^{\infty}\left|Q\left(B \beta^{k-n}\right)\right|=C \prod_{n=1}^{k}\left|Q\left(B \beta^{n}\right)\right| \geqslant C \prod_{n=1}^{\infty}\left|Q\left(B \beta^{n}\right)\right| .
$$

Now we recall a well-known property of the PV number [27]: there exists $0<\theta<1$ such that $\left|\left[\beta^{n}\right]\right|<\theta^{n}$ for large $n$, where $[x]$ denotes the distance from $x$ to the nearest integer. We can choose $n_{0}$ such that for $n>n_{0}, 2 B_{j} \theta^{n}<\frac{1}{2}$. Let $C^{\prime}=\prod_{n=1}^{n_{0}}\left|Q\left(B \beta^{n}\right)\right|>0$. Then

$$
\begin{aligned}
\prod_{n=1}^{\infty}\left|Q\left(B \beta^{n}\right)\right| & =C^{\prime} \prod_{n=n_{0}+1}^{\infty}\left|Q\left(B \beta^{n}\right)\right| \\
& =C^{\prime} \prod_{n=n_{0}+1}^{\infty}\left|\sum_{j=1}^{N} w_{j} e^{2 \pi i B_{j}\left[\beta^{n}\right]}\right| \\
& \geqslant C^{\prime} \prod_{n=n_{0}+1}^{\infty} \min _{1 \leqslant j \leqslant N}\left|\operatorname{Re} e^{2 \pi i B_{j} \theta^{n}}\right| \\
& \geqslant C^{\prime} \prod_{1 \leqslant j \leqslant N} \prod_{n=n_{0}+1}^{\infty} \cos \left(2 \pi B_{j} \theta^{n}\right) .
\end{aligned}
$$

The product is a positive constant and if we put it back into expression (5.3), we have $\Phi\left(B \beta^{k}\right) \nrightarrow 0$ as $k \rightarrow \infty$. 
Note that in the proof we have not used the property of the positive weights. The proof can hence be applied directly to conclude the following corollary.

COROLlary 5.1. Let the IFS be as in Theorem 5.1, and let $\sum_{j=1}^{N} c_{j}=\rho^{-1}$. Then the functional equation

$$
f=\sum_{j=1}^{N} c_{j} f \circ S_{j}^{-1}
$$

has no $L^{1}$-solution.

Acknowledgements. The authors would like to thank Dr Lifeng Xi for the simplification of Lemma 5.1 from the original, more complicated, argument. The second and third authors acknowledge their gratitude to the Department of Mathematics, Chinese University of Hong Kong, for postdoctoral support. The second author also acknowledges the support received from the Southeast Applied Analysis Center in the School of Mathematics, Georgia Institute of Technology.

\section{References}

1. J. C. Alexander and J. A. Yorke, 'Fat baker's transformations', Ergodic Theory Dynam. Systems 4 (1984) $1-23$.

2. J. C. AleXAnder and D. Zagier, 'The entropy of a certain infinitely convolved Bernoulli measure', J. London Math. Soc. 44 (1991) 121-134.

3. C. BAndt, 'Self-similar sets 5. Integer matrices and fractal tilings of $\mathbb{R}^{n}$, Proc. Amer. Math. Soc. 112 (1991) 549-562.

4. C. BANDT and S. GRAF, 'Self-similar sets 7. A characterization of self-similar fractals with positive Hausdorff measures', Proc. Amer. Math. Soc. 114 (1992) 995-1001.

5. A. FAN, K. S. LAU and S. M. NGAI, 'Iterated function systems with overlaps', Asian J. Math. 4 (2000) 527-552.

6. A. M. Garsia, 'Arithmetic properties of Bernoulli convolutions', Trans. Amer. Math. Soc. 102 (1962) $409-432$.

7. G. H. Hardy and J. E. LitTLEwood, 'Some properties of fractional integrals I', Math. Z. 27 (1928) 565-606.

8. T. Hu, 'The local dimensions of the Bernoulli convolution associated with the golden number', Trans. Amer. Math. Soc. 349 (1997) 2917-2940.

9. T. Hu and K. S. LAU, 'Multifractal structure of convolution of the Cantor measure', Adv. in Appl. Math. (to appear).

10. J. E. Hutchinson, 'Fractals and self similarity', Indiana Univ. Math. J. 30 (1981) 713-747.

11. M. Keane, M. Smorodinsky and B. Solomyak, 'Morphology of $\gamma$-expansions with deleted digits', Trans. Amer. Math. Soc. 347 (1995) 955-966.

12. R. Kenyon, 'Projecting the one-dimensional Sierpinski gasket', Israel J. Math. 97 (1997) 221-238.

13. J. Lagarias and Y. Wang, 'Self-affine tiles in $\mathbb{R}^{n}$ ', Adv. Math. 121 (1996) 21-49.

14. S. P. Lalley, ' $\beta$-expansions with deleted digits for Pisot numbers', Trans. Amer. Math. Soc. 349 (1997) 4355-4365.

15. K. S. LAU, 'Dimension of a family of singular Bernoulli convolutions', J. Funct. Anal. 116 (1993) 335-358.

16. K. S. LAU and S. M. NGaI, ' $L^{q}$-spectrum of the Bernoulli convolution associated with the golden ratio', Studia Math. 131 (1998) 225-251.

17. K. S. LAU and S. M. NGaI, 'Multifractal measures and a weak separation condition', Adv. Math. 141 (1999) 45-96.

18. K. S. LAU and S. M. NGaI, ' $L^{q}$-spectrum of Bernoulli convolutions associated with the PV numbers', Osaka J. Math. 36 (1999) 993-1010.

19. K. S. LAU and J. WANG, 'Mean quadratic variations and Fourier asymptotics of self-similar measures', Monatsh. Math. 115 (1993) 99-132.

20. K. S. LAU, M. F. MA and J. WANG, 'On some sharp regularity estimation of $L^{2}$-scaling functions', SIAM J. Math. Anal. 27 (1996) 835-864.

21. F. LedrapPier and A. Porzio, 'A dimension formula for Bernoulli convolutions', J. Statist. Phys. 76 (1994) 1307-1327.

22. Y. PERES and W. SCHLAG, 'Smoothness of projections, Bernoulli convolutions and the dimension of exceptions’, Duke Math. J. 102 (2000) 193-251. 
23. Y. Peres and B. Solomyak, 'Absolute continuity of Bernoulli convolutions: a simpler proof', Math. Res. Lett. 3 (1996) 231-239.

24. Y. Peres and B. Solomyak, 'Self-similar measures and intersection of Cantor sets', Trans. Amer. Math. Soc. 350 (1998) 4065-4087.

25. M. Pollicott and K. Simon, 'The Hausdorff dimension of $\lambda$-expansions with deleted digits', Trans. Amer. Math. Soc. 347 (1995) 967-983.

26. H. RAO and Z. Y. WEN, 'Some studies of a class of self-similar fractals with overlap structure', Adv. in Appl. Math. 20 (1998) 50-72.

27. R. SALEM, Algebraic numbers and Fourier analysis, Heath Mathematical Monographs (Heath, Boston, 1962).

28. B. SolomyaK, 'On the random series $\sum \pm \lambda^{n}$ (an Erdös problem)', Ann. of Math. 142 (1995) 611-625.

29. R. S. Strichartz, 'Self-similar measures and their Fourier transforms III', Indiana Univ. Math. J. 42 (1993) 367-411.

30. Y. Xu, 'Fractal $n$-hedral tilings of $\mathbb{R}^{d}$, J. Austral. Math. Soc. 66 (1999) 403-417.

K. S. Lau

Department of Mathematics

Chinese University of Hong Kong

Hong Kong

kslau@math.cuhk.edu.hk

H. Rao

Department of Mathematics

Wuhan University

Wuhan 430072

China

raohui@nlsc.whu.edu.cn
S. M. Ngai

School of Mathematics

Georgia Institute of Technology

Atlanta

GA 30332

USA

Current address of S. M. Ngai: Department of Mathematics Georgia Southern University

Statesboro

GA 30460

USA

ngai@gsu.cs.gasou.edu 